\title{
Reconhecimento de Estados Afetivos do Aluno em Ambientes Virtuais de Aprendizagem
}

\author{
Magalí Teresinha Longhi \\ Magda Bercht \\ Patricia Alejandra Behar \\ Programa de Pós-Graduação em Informática na Educação \\ Universidade Federal do Rio Grande do Sul (UFRGS) \\ Caixa Postal 5071 - 90.041-970 - Porto Alegre - RS - Brasil \\ mlonghidcesup.ufrgs.br, berchteinf.ufrgs.br, pbehareterra.com.br
}

Resumo: O presente estudo aborda a incorporação de dimensões afetivas em ambientes virtuais de aprendizagem a partir da identificação dos estados de ânimo do aluno, de modo a favorecer estratégias pedagógicas mais adequadas. A partir da revisão teórica acerca do reconhecimento de estados afetivos, apresenta-se o protótipo AnimA, direcionado à inferência de diferentes estados de ânimo durante o processo de aprendizagem. O protótipo considera as interações do aluno com seus colegas e com o professor através das ferramentas de comunicação do ambiente virtual de aprendizagem ROODA.

Palavras-chave: reconhecimento de estados afetivos, estados de ânimo, computação afetiva, ambientes virtuais de aprendizagem.

\section{Identification of Students' Emotional States in Virtual Learning Environments}

Abstract: The present study is about the incorporation of the emotional dimension in virtual learning environments following the identification of students' mood so as to encourage a more adequate pedagogical practice. After a theoretical revision about mood recognition, we present the prototype AnimA, aimed at the inference of different emotional states during the learning process. The prototype considers the interactions of students with their peers and teachers through the communication tools from the learning environment ROODA.

Key-words: affective recognition, mood, affective computing, virtual learning environments.

\section{Introdução}

A pesquisa da Inteligência Artificial aplicada na Educação tem se mostrado muito ativa. Os atuais processos de ensino e aprendizagem humana e o advento de novas tecnologias para implantação de salas de aulas e mundos virtuais ensejaram uma nova interação social mediada por computadores. Disto deriva uma nova abordagem para os ambientes virtuais de aprendizagem (AVA) ${ }^{(1)}$. A avaliação do processo de ensino e aprendizagem deve ir além da verificação do atingimento dos objetivos em relação ao conteúdo, levando em consideração o afeto e os fenômenos afetivos subjacentes do aluno em interação, visto que eles interferem profundamente nos processos mentais como memorização, raciocínio, atenção, motivação, etc. (Picard et al., 2004; Bercht, 2001; Damásio, 2000; Piaget, 1978; Vygotski, 2001; Wallon, 1971).

Os termos: emoção, estados de humor/ânimo, motivação, sentimento, paixão, personalidade, temperamento e outros tantos estão relacionados à afetividade; já os termos: razão, raciocínio, percepção, memória, compreensão, atenção, juízo, pensamento, linguagem, bom-senso e inteligência estão relacionadas à cognição. A definição de cada um deles ainda 
provoca confusão, já que as dimensões afetivas e cognitivas são estudadas em áreas de conhecimento diversas, e não em âmbito interdisciplinar.

Neste trabalho, o termo afetividade é utilizado para identificar um conjunto de fenômenos psíquicos e físicos que incluem o domínio das emoções propriamente ditas, dos sentimentos das emoções, das experiências sensíveis e, principalmente da capacidade em se poder entrar em contato com sensações (Bercht, 2001 p.59).

O termo cognição é ainda empregado para identificar o conjunto de processos mentais que participam na aquisição de conhecimento, na percepção do mundo (e de nós mesmos) e de como este mundo é representado. Entretanto, descobertas da neurociência indicam que cognição e afetividade são elementos indissociáveis. Uma redução ou excesso de afetividade resulta em problemas na cognição. E esta não se sobrepõe à afetividade. Pelo contrário, a afetividade pode auxiliar no raciocínio, principalmente em questões pessoais e sociais que envolvam conflitos, revelando sua importância na tomada de decisões (Damásio, 2000) e, assim como, nos processos de aprendizagem.

Becht (2001) sugere que a integração dos fenômenos afetivos a sistemas computacionais os transformam em sistemas adaptativos e funcionalmente eficientes nas interações humanas mediadas por computador. Deste modo, torna-se importante o estudo da afetividade nas interações realizadas através de AVA. Behar et alli (2005) enfatizam que esses ambientes são apresentados como sistemas abertos, onde os participantes estão em constante interação e evolução, atuando, ao mesmo tempo, num espaço de ordem e desordem, de cooperação e colaboração, princípios fundamentais no processo aprendizagem.

Para tanto, busca-se incorporar dimensões afetivas no ambiente virtual de aprendizagem ROODA ${ }^{(2)}$ (Rede cOOperativa De Aprendizagem) através do reconhecimento dos estados de ânimo do aluno, de modo a favorecer ações pedagógicas que permitam ao professor a tomada de decisão baseadas em aspectos afetivos inferidos.

Na próxima seção, procede-se a uma breve revisão teórica acerca da importância da afetividade na subjetividade e como estes estudos vêm sendo incorporados em sistemas educacionais informatizados. Na seção 3, é relatado o estado-da-arte das abordagens no que diz respeito ao reconhecimento dos fenômenos afetivos. Na seção 4, é apresentado o ambiente ROODA. Na seção 5, descreve-se o protótipo AnimA em desenvolvimento para o reconhecimento dos estados de ânimo de alunos durante execução de uma atividade de resolução de problemas computacionais aplicada em alunos da graduação do curso Ciência da Computação, enquanto na seção 6, a experimentação do protótipo é abordado. O AnimA é suporte para outras experiências a serem desenvolvidas com alunos de graduação de outros cursos. O resultado destes experimentos servirá de subsídio para determinar a possibilidade do reconhecimento dos estados de ânimo durante a realização de tarefas de aprendizagem. Finalmente, na última seção, são apresentadas algumas considerações e abordado o direcionamento de pesquisa.

\section{Afetividade e Dimensões Afetivas na Ciência da Computação}

Até o início do século XX, os movimentos filosóficos impulsionaram os debates sobre pensamento, conhecimento, comportamento, razão e emoção. Mais tarde, com a consolidação das grandes teorias psicológicas - gestalt, psicanálise, behaviorismo, epistemologia genética, psicologia cultural e sócio-histórica -, a importância da afetividade nas atividades cognitivas passou ser reconhecida.

A psicogenética construtivista tem contribuído para desmitificar a dicotomia entre afetividade e cognição. Piaget (1962) reconheceu que a afetividade é o agente motivador da atividade cognitiva: emoção e razão constituiriam termos complementares. Vygotski (2001) concebia a linguagem e a interação como elementos fundamentais da consciência e do 
aprendizado. Questionou o dualismo entre as dimensões afetivas e cognitivas, ao assinalar que a psicologia tradicional pecava em separar os aspectos intelectuais dos afetivos-volitivos. Por sua vez, Wallon (1971) viria a postular que afetividade e inteligência apresentam-se misturadas: a consciência afetiva dá origem à atividade cognitiva. $E$ que, durante $o$ desenvolvimento humano, existem fases em que predominam o afeto; noutras, a inteligência.

Com a evolução da neurociência (Damásio, 1996; Le Doux, 2005) tornou-se possível demonstrar que os processos cognitivos e afetivos são indissociáveis, de modo que o conjunto de fenômenos afetivos exercem influência nos processos mentais e os sistemas cerebrais destinados à afetividade apresentam-se intrinsecamente ligados aos sistemas destinados à cognição. Entre tantos outros pesquisadores das ciências cognitivas, Ortony et alli (1988), Roseman et alli (1990), Izard (1993), Scherer (2005) concordam que crenças e desejos influenciam a ação, que a interação com outros e com o ambiente pode originar novas crenças e que, por sua vez, novas necessidades podem provocar novos desejos. Dessa forma, crenças, desejos e intenções exercem influências sobre os processos cognitivos, os quais, por sua vez, ativam dimensões afetivas no sujeito (e vice-versa). Sendo assim, a afetividade tem papel importante no comportamento inteligente, na tomada de decisão, na comunicação social, processos estes que supõem habilidades racionais.

Os estados de ânimo ${ }^{(3)}$ constituem fenômenos afetivos diferenciados das emoções ${ }^{(4)}$ por serem globais, difusos, de longa duração e constantes. Ao contrário, convém destacar, o estado de ânimo é um estado afetivo de baixa intensidade, às vezes com pouca sincronização de respostas que podem permanecer por horas ou dias (Scherer, 2005; Sloman, 2002), nem sempre desencadeado por eventos externos. Scherer (2005) classifica como estados de ânimo as categorias: 1) animado (no sentido de estar disposto, satisfeito, esperançoso, alegre, entusiasmado); 2) desanimado (estar triste, abatido, deprimido, pessimista, desgostoso, melancólico); 3) indiferente (desatento); 4) ansioso (preocupado, aflito); e 5) irritado (impaciente).

Apesar dos avanços proporcionados pelas teorias biológicas, psicológicas, sociológicas no sentido de compreender a afetividade e do reconhecimento de sua importância na construção social, a Ciência da Computação, através da Inteligência Artificial, não a tinha como relevante para fins de aplicação em agentes inteligentes ${ }^{(5)}$. Somente no final dos anos 90 , com a introdução do conceito de Computação Afetiva por Picard (1997), passou-se a considerar a afetividade em sistemas computacionais. Tal conceito discutia a aplicabilidade da afetividade em sistemas não-biológicos, ou seja, o uso das emoções em diferentes aspectos nos sistemas computacionais, desde o reconhecimento, representação e simulação até pesquisas relacionadas aos diferentes fenômenos afetivos que se verificam nas interações homemmáquina.

Atualmente, os poucos sistemas educativos informatizados que propõem tratar a dimensão afetiva tem como foco as emoções. Pouca atenção tem sido dada aos estados de ânimo do aluno no processo de aprendizagem. Do conjunto de emoções, apenas as conhecidas como básicas $^{(6)}$ (a saber: raiva, medo, alegria, tristeza, nojo, vergonha e culpa) costumam ser aplicadas. Elas podem ser inferidas por informações como gestos, expressões faciais, linguagem (textual, oral e de sinais), tensão muscular, condutividade da pele, respiração, ritmo cardíaco, temperatura, movimentos oculares, e pelo comportamento observável (Picard et al., 2004). O uso de equipamentos com sensores visuais (video-câmeras), de áudio (microfones) e fisiológicos (cadeiras sensíveis à pressão do corpo, luvas que captam a condutividade da pele, mouse sensível à "qualidade" de pressão, EEG, ECG, ERP, termógrafos, aparelhos para verificar pulsação, respiração, dilatação da pupila) ainda são onerosos e de difícil utilização, além de desconfortáveis e passíveis de interferir no reconhecimento das emoções.

Pesquisas empreendidas na Computação Afetiva voltadas para a Informática na Educação envolvem a construção de agentes afetivos pedagógicos (Paiva \& Machado, 1998; 
Rickel \& Johnson, 1998; Shaw et al., 1999; Jaques \& Vicari, 2005) em sistemas tutores inteligentes ${ }^{(7)} \mathrm{em}$ que as emoções são reconhecidas através do comportamento observável do aluno. A análise é feita aplicando-se modelos computacionais baseados na Teoria do Appraisal, que avalia os eventos antecedentes aos estados afetivos. Os frameworks que tratam dos estados de ânimo examinam apenas o estado bipolar: humor positivo ou negativo (Rousseau \& Hayes-Roth, 1998; Kshirsagar \& Magnenat-Thalmann, 2002).

Apesar dos estudos envolvendo a incorporação de emoções e da tentativa de incluir estados de ânimo em agentes, vários desafios ainda se apresentam no reconhecimento e expressão da afetividade na construção de interfaces homem-máquina. Sem dúvida, o reconhecimento e a expressão da afetividade são elementos essenciais na comunicação e estão estritamente relacionados a temas como: estar ou não motivado, estar ou não aborrecido, estar ou não indiferente, estar ou não frustrado, etc., fatores importantes a considerar no processo de aprendizagem (por exemplo, ao favorecer a intervenção dos professores, quando se fizer necessário).

\section{Abordagens para reconhecimento de estados afetivos}

Um dos pressupostos da Computação Afetiva é o de que os computadores tenham a capacidade de reconhecer e de inferir aspectos afetivos como se fossem observadores em terceira-pessoa (Picard, 1997). Desse modo, podem entender as formas de expressão afetiva humana e, quando necessário, sintetizá-la.

Os fenômenos afetivos, tanto quanto os pensamentos, são expressos, voluntária ou involuntariamente, na forma de sons (palavras faladas, cantadas, cantaroladas ou deixas), de grafia (palavras escritas, desenhos, pinturas, etc.), de gestos (faciais, corporais), de comportamento (agitação, tranquilidade, rubor, etc.) e de outras formas criativas para exprimir a comunicação. A comunicação afetiva é captada por meio dos sentidos em ambiente real ou através de instrumentos mediadores (papel, telefone, vídeo, etc.). Pode ser visível ou subentendida. Em quaisquer dos casos, a comunicação afetiva é feita através de um padrão de informação que pode ser representado no computador. O reconhecimento dos estados afetivos passa a constituir um problema de reconhecimento de padrões (Picard, 1997).

Os métodos de inferência dos aspectos afetivos apresentadas na literatura (Liao et al., 2006) podem ser classificados em inferência por prognóstico (top-down), inferência por diagnóstico (botton-up) e inferência híbrida (combinação de prognóstico e diagnóstico). No primeiro caso, o reconhecimento dos aspectos afetivos é baseado em fatores que influenciam ou causam o estado afetivo. Esta abordagem é apoiada por teorias psicológicas. Um exemplo é a teoria OCC (Ortony et al., 1988), largamente utilizada para sintetizar 22 estados afetivos conforme apresentado em Bercht (2001) e Jaques \& Vicari (2005), onde um estado afetivo é determinado a partir da sua avaliação sob três aspectos: consequiência dos eventos, ação dos agentes envolvidos na comunicação afetiva e aparência dos objetos envolvidos nesta comunicação. As percepções afetivas são valoradas a partir de seus objetivos, padrões e preferências. O cálculo da intensidade do estado afetivo reconhecido é derivado a partir de variáveis globais (senso de realidade, proximidade, etc.) e locais (probabilidade do evento ocorrer, esforço para atingir o objetivo, possibilidade da realização do objetivo, etc.).

Na segunda abordagem, inferência por diagnóstico, o reconhecimento é feito através de medidas comportamentais e fisiológicas. Vários trabalhos estão sendo desenvolvidos para capturar padrões afetivos. Kapoor e Picard (2005) apresentam uma proposta multi-sensorial para reconhecer e avaliar o interesse (ou desinteresse) de uma criança durante um jogo educacional por computador. Para tanto, são obtidas informações sobre as expressões faciais e movimentos da cabeça, como também a postura dos alunos nas cadeiras. Zeng et alli (2004) e Ji \& Hu (2002) extraem características afetivas das feições através de imagens de vídeos, e 
Kapur et alli (2005) utilizam a técnica de motion capture $^{(8)}$ para capturar os movimentos corporais nos diferentes estados afetivos. Scherer (1995) usa as teorias socio-pscicológicas para explicar e determinar o potencial emocional da voz durante a fala e o canto. O agente pedagógico PAT (Jaques \& Vicari, 2005) infere as emoções do aluno em função de seu comportamento observável, isto é, pelas ações do aluno na interface do sistema (por exemplo: tempo de execução de uma atividade, sucesso ou falha na execução de um exercício, pedido de ajuda, etc.).

Os métodos de prognóstico e diagnóstico podem ser combinados, originando a abordagem híbrida, cujo resultado são inferências afetivas mais precisas do reconhecimento realizado. Conati \& Maclaren (2004) recorrem a métodos probabilísticos para combinar traços de personalidade com métodos de diagnóstico através de sensores biométricos. Já Liao et alli (2006) concebem um sistema integrado para reconhecer, em tempo real, os estados afetivos fadiga e estresse combinando (a) inferências probabilísticas obtidas pelas evidências geradas nas diversas modalidades de captura de sinais afetivos, (b) sensores para identicar no tempo as medidas dos sinais afetivos do usuário (medidas fisiológicas, comportamental, de performance e aparência física), e (c) determinação da necessidade de assistência ao usuário em estado de estresse ou fadiga.

Percebe-se que a maioria dos trabalhos da literatura prende-se a uma ou outra abordagem (inferência por prognóstico ou diagnóstico). Neste trabalho, utiliza-se a abordagem híbrida para o reconhecimento dos estados de ânimo. Esta é caracterizada, inicialmente, pela captura de medidas comportamentais, auto-registradas e observáveis, e pela inferência probabilísitica obtida pelas evidências geradas nas três modalidades listadas.

\section{Ambiente virtual ROODA}

O ROODA tem como meta principal a mudança de paradigma educacional a partir da interação e cooperação dos usuários em AVA, sendo, por isso, centrado no usuário e orientado a valorizar o processo de cooperação. Para Behar et alli (2005), o objetivo dessa plataforma é oferecer possibilidades através de recursos na web, para que os usuários (professores, monitores e alunos) construam um trabalho cooperativo através de interações virtuais e sociais, transformando seu modo de pensar a partir da convivência e da troca entre alunos e professores. O diferencial deste ambiente, conforme Behar et alli (2007), reside em uma plataforma multi-cursos centrada no aluno, evitando que tenha que se conectar ao ambiente cada vez que vai para um curso, disciplina ou turma diferente.

Os conceitos-chave relevantes que direcionaram o projeto pedagógico do ambiente ROODA são: 1) a aprendizagem, destacando-a como uma mudança estrutural que ocorre na convivência, no encontro entre os usuários; 2) a troca/interação, que se tornam indispensáveis para a construção de conhecimento; 3) a cooperação, a qual pode promover conflitos sóciocognitivos dentro de um grupo (pensar no ponto de vista de outro, se perturbar, descentrar, se equilibrar e re-equilibrar); 4) a autonomia do usuário, de modo a que possa avaliar o resultado de suas escolhas, reforçando o seu papel de agente ativo no processo de aprendizagem; 5) a liberdade de acesso, no sentido de que todos estão autorizados a dispor de toda produção disponível no ambiente; 6) a avaliação, a ser realizada durante todo o processo de aprendizagem, com o objetivo de acompanhar e orientar o aluno; 7) a participação do aluno no processo de avaliação; 8) a não-planificação dos objetivos a serem atingidos pelos alunos.

O ROODA, reconhecido institucionalmente pela UFRGS em 2003 como ambiente de EAD, é o AVA escolhido neste trabalho como plataforma de aplicação do framework de reconhecimento e validação dos estados de ânimo dos alunos durante os processos de interação e contrução do conhecimento. 
A base para os estudos dos estados de ânimo no ambiente ROODA compreende: a) os textos produzidos pelos alunos ao utilizarem os recursos de interação síncrona (bate-papo e A2) e assíncrona (fórum e diário de bordo), por representarem as interações no ambiente virtual; e b) a funcionalidade InterROODA, a qual registra os acessos qualitativos e quantitativos individuais e entre os alunos.

\section{Protótipo para o Reconhecimento e Modelagem dos Estados de Ânimo}

O protótipo AnimA tem por finalidade servir de apoio para a validação do reconhecimento e de alguns estados de ânimo envolvidos nas interações entre os alunos de graduação. Este projeto é um experimento para validar os parâmetros necessários no desenvolvimento da ferramenta ROODAfeto que indicará aos formadores e professores dos cursos a visualização da motivação inferida do aluno durante a interação do mesmo no ambiente ROODA.

O protótipo considera as seguintes categorias afetivas, baseadas nas definições de Scherer (2005), para os estados de ânimo: (1) estar animado - implica demonstrar um comportamento alegre, boa disposição, motivação, interesse, satisfação para enfrentar os desafios da aprendizagem, e, colabora e coopera com os colegas; (2) estar desanimado implica demonstrar um descontentamento, comportamento triste, sem disposição, desinteresse, sem motivação, insatisfação, frustração (ou sentir-se penalizado) para continuar o aprendizado; ou ainda, sentir-se coagido, por acreditar que a vontade do colega prevalece; (3) estar indiferente - implica demonstrar apatia, displicência, negligência, descaso e falta de motivação pelos conteúdos da aprendizagem.

O reconhecimento dos estados de ânimo no protótipo AnimA envolve quatro etapas (Figura 1, inspirada em Liao et alli, (2006)): a) aquisição e identificação; b) interpretação; c) seleção e d) inferência dos estados de ânimo do aluno. A aquisição e identificação determinam os meios e os métodos através dos quais o sistema reconhecerá características referentes aos estados afetivos em análise. Para tanto, pode-se utilizar indicadores aparentes (expressão facial, entonação de voz, gestos corporais, postura, etc.), semi-aparentes (expressão textual), não-aparentes (respiração, batimentos cardíacos, pressão sanguínea, temperatura corporal, etc.) e de avaliação (inventários, questionários, etc.). Estes sinais passam por sistema de reconhecimento de padrões (de fala, de escrita, de faces, de sinais, de dados, etc) submetidos a modelos estatísticos e a processamento de imagens e de dados. A interpretação diz respeito à análise automática (ou não) das informações extraídas pelos métodos de reconhecimento de padrões. A etapa de seleção filtra os dados importantes para o motor de inferência dos estados de ânimo do aluno.

Em sua primeira versão, o AnimA reconhece os estados de ânimo considerando os indicadores semi-aparentes e os de avaliação, os modelos estatísticos de linguagem para identificação dos estados de ânimo na escrita e, finalmente, o tratamento de dados para classificação e categorização dos mesmos advindos dos inventários e questionários de pesquisa.

Os indicadores semi-aparentes são obtidos no ambiente ROODA, podendo os estados de ânimo do aluno ser observados em dois cenários: (1) O aluno interage com o conteúdo, exercícios e atividades não-colaborativas do ambiente: o sistema baseia-se nas ações do aluno (acertar ou errar um exercício ou tarefa; tempo passado no ambiente; demora em efetivar uma resposta, etc) para inferir os estados de ânimo e estabelecer que ações pedagógicas aplicar; (2) expressa suas idéias e colabora com colegas através das ferramentas de comunicação assíncronas, a exemplo do fórum e do diário de bordo. 


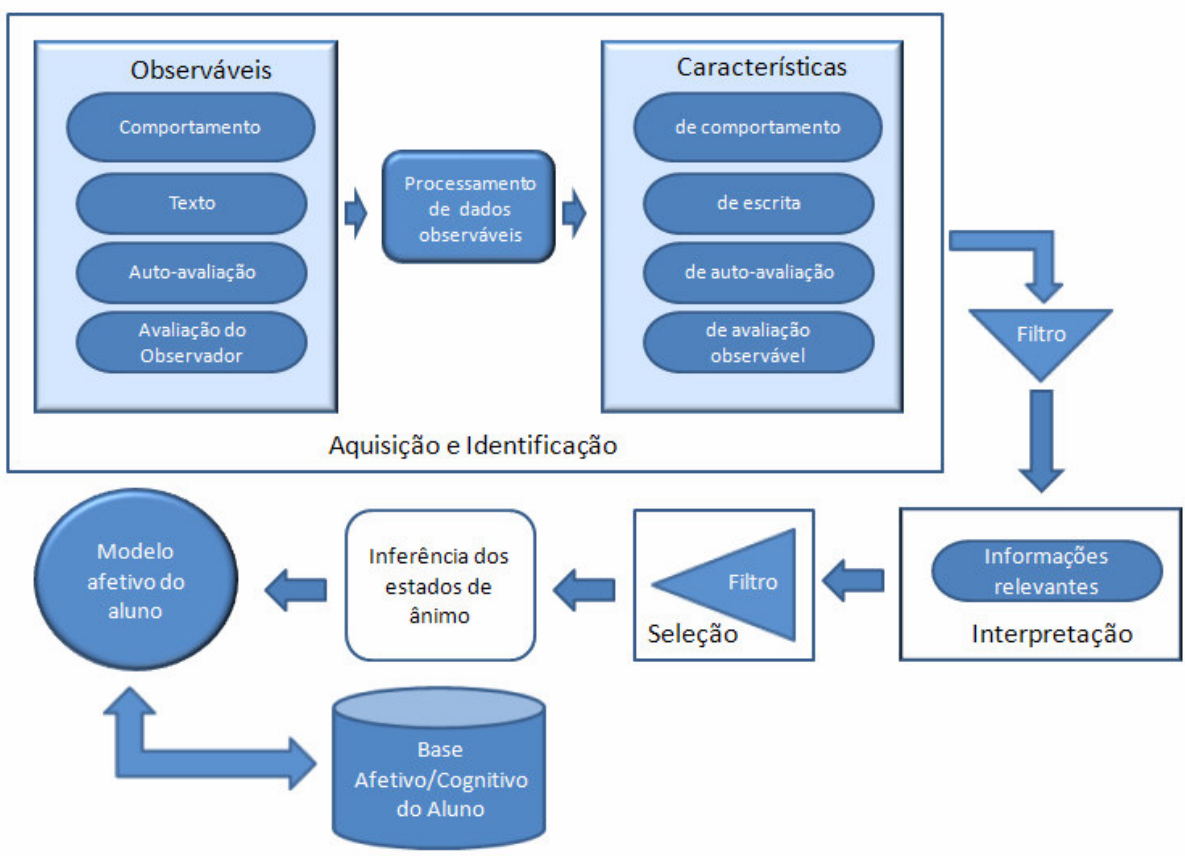

Figura 1: Esquema geral do reconhecimento dos estados de ânimo

Em todos os cenários acima, considerar a afetividade do usuário envolve duas importantes etapas: (1) a inferência dos estados de ânimo do aluno e (2) a determinação do modelo do aluno essenciais para a discussão e determinação das táticas afetivas a serem implementadas na ferramenta ROODAfeto.

\section{O Experimento}

O domínio construção de algoritmos computacionais foi escolhido como atividade do protótipo AnimA para os alunos do curso de Ciência da Computação. Os participantes deverão entender o problema da troca de lugares entre sapos machos e fêmeas (considerando as regras impostas para tal), encontrar uma metodologia para solucionar o problema e formular o algoritmo que poderá ser reescrito posteriormente em linguagem computacional. O projeto da interface pode ser visto na figura 2 (inspirada em Vicente \& Pain (2002)).

Em diversos momentos de desenvolvimento da atividade (início, etapas intermediárias, e finalização do algoritmo), o sistema convidará o aluno para registrar as percepções de seus estados de ânimo em janela à parte, seu processo de aprendizagem e suas considerações/colaborações nas ferramentas Diário de Bordo e Fórum do ambiente ROODA.

O instrumental metodológico abrange tanto a auto-avaliação do aluno quanto a avaliação do sistema e a dos observadores. No início do semestre são aplicados instrumentos de auto-avaliação e avaliação observada. Durante a apresentação, desenvolvimento e finalização da atividade, os alunos realizam a auto-avaliação do processo, enquanto o sistema faz sua própria inferência e os observadores as suas. No final do semestre, a auto-avaliação e a avaliação observada são reaplicadas.

Os instrumentos de auto-avaliação e avaliação observada do início e final do semestre são inventários disponibilizados no ambiente ROODA. A avaliação do sistema é baseada na captura de medidas comportamentais considerando-se as variáveis: confiança, esforço, independência (conforme Bercht (2001)), satisfação e desafio (conforme Vicente \& Pain (2002)) durante o desenvolvimento da atividade; e cooperação, colaboração, e coação (conforme Macedo (2005)) durante as interações sociais. 
O resultado destas investigações será subsídio para o desenvolvimento da ferramenta ROODAfeto, que delineará as ações pedagógicas-afetivas referentes ao comportamento do aluno durante as atividades de aprendizagem.

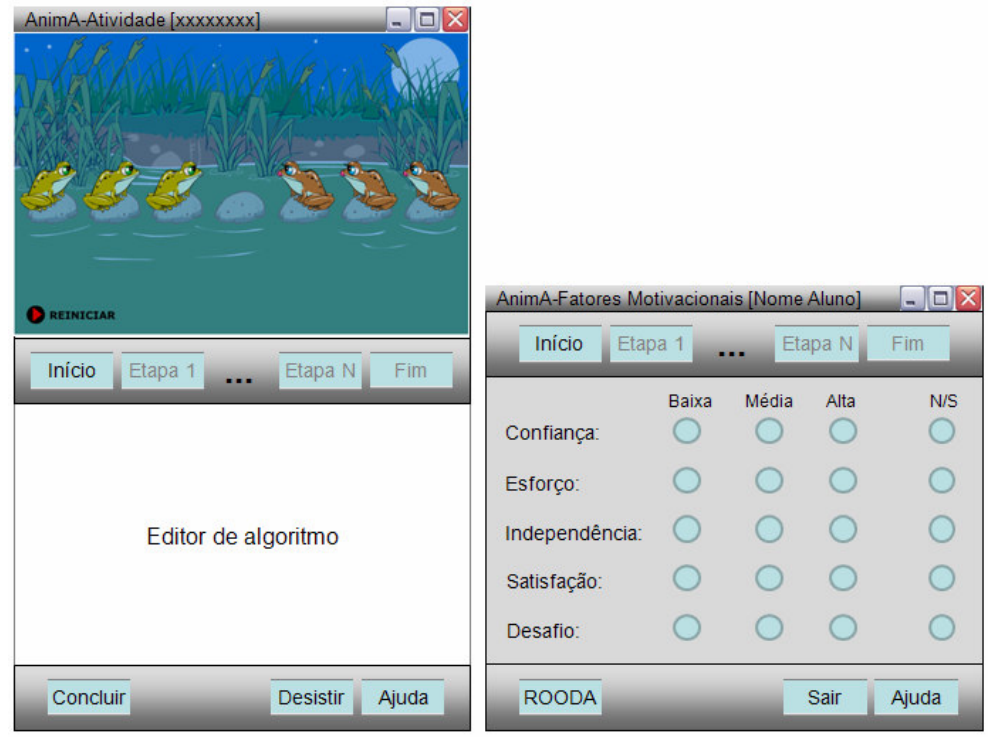

Figura 2: Projeto de interface do AnimA

\section{Considerações finais}

É crescente o interesse no desenvolvimento de ferramentas tecnológicas direcionadas a sistemas educacionais que tratam do reconhecimento de fenômenos afetivos. O desafio, agora, é o de desenvolver instrumentos que, partindo do reconhecimento dos estados afetivos do aluno, permitam ao professor uma percepção mais acurada a respeito de sua motivação.

Discute-se, neste trabalho, a introdução de dimensões afetivas em ambientes virtuais de aprendizagem a partir do reconhecimento de seus estados de ânimo, visando favorecer ações pedagógicas que permitam ao professor a tomada de decisões baseadas em aspectos afetivos inferidos durante os processos de interação com o sistema. Para tanto, é apresentado o protótipo AnimA que se vale da abordagem híbrida - inferências por prognóstico e por diagnóstico.

Pretende-se aplicar os experimentos durante o semestre de 2008/I em discentes do curso Ciência da Computação e, posteriormente, em alunos de Pedagogia e em cursos de diferentes áreas. O estudo é relevante para determinar se as variáveis escolhidas para o delineamento das categorias de estado de ânimo devem ser consideradas (ou reavaliadas) quando do desenvolvimento da ferramenta ROODAfeto.

\section{Notas de Texto}

(1) Um AVA é constituído por uma infra-estrutura tecnológica (interface gráfica, comunicação síncrona/assíncrona e outras funcionalidades) e por todas as relações (afetivas, cognitivas, simbólicas, entre outras) estabelecidas pelos sujeitos participantes, tendo como foco principal a aprendizagem (Behar et al., 2005).

(2) Disponível online no endereço http://www.ead.ufrgs.br/rooda.

(3) Na língua portuguesa, humor pode ser entendido como uma substância orgânica líquida ou semilíquida; a capacidade que o sujeito possui para perceber, apreciar ou expressar o que é 
cômico ou divertido; ou seu estado de humor no sentido de disposição de espírito/ânimo. Neste trabalho, usa-se o termo estado de ânimo como sendo o estado de humor baseado na disposição do espírito do sujeito.

(4) A emoção é um episódio relativamente breve, de resposta sincronizada na avaliação de um evento interno ou externo (Scherer, 2005).

${ }^{(5)}$ Entende-se como agente inteligente um sistema computacional que realiza ações racionais de forma autônoma e aprende ao interagir com o seu entorno. Também tem como características a capacidade de solucionar problemas e a de cooperar com outros agentes, configurando sistemas multi-agentes.

(6) Scherer (2005) descreve o fenômeno afetivo como o conjunto: das preferências, das atitudes ou posturas, dos estados de humor (ou ânimo), das disposições afetivas, das posturas interpessoais, das emoções estéticas e das emoções utilitárias. O conjunto de emoções utilitárias coincide com o conjunto de emoções básicas propostas por Ekman (1999).

(7) Sistemas tutores inteligentes são programas que usam de técnicas de IA e possuem a capacidade de aprender e adaptar as estratégias de ensino de acordo com o desenrolar do diálogo com o aluno (Bercht, 2001).

(8) Motion capture é uma técnica usada para capturar e registrar digitalmente os movimentos do corpo e face através de sensores aplicados em regiões próximas às juntas ou pontos de grande movimentação dos músculos.

\section{Referências Bibliográficas}

Behar, P.A.; Walquil, M.; Bernardi, M \& Moresco, S. Refletindo sobre uma metodologia de pesquisa para AVA's.In: Congresso Internacional de Qualidade de Educação a Distância, 2005, São Leopoldo. Anais. São Leopoldo: Unisinos, v. 1. 2005.

Behar, P. A.; Leite, S. M.; Bordini, S.; Souza, L. B.; Siqueira, L. G.. Avaliação de Ambientes Virtuais de Aprendizagem: O Caso do ROODA na UFRGS. In: Revista Avances en Sistemas e Informática, v. 4, p. 81-100, Bogatá, 2007.

Bercht, M.. Em Direção a Agentes Pedagógicos com Dimensões Afetivas. Instituto de Informática. UFRGS. Tese de Doutorado. 2001.

Conati, C., \& Maclaren, H. Evaluating a Probabilistic Model of Student Affect, ITS'04, International Conference on Intelligent Tutoring Systems, Maceio, Brazil. 2004.

Damásio, A. O Erro de Descartes: emoção, razão e o cérebro humano. Tradução: Dora Vicente e Georgina Segurado. São Paulo: Cia das Letras, 1996.

Damásio, A. O Mistério da Consciência: do corpo e das emoções ao conhecimento de si. Tradução: Laura Teixeira Motta. São Paulo: Cia das Letras, 2000.

Ekman, P.. Basic Emotions In: T. Dalgleish and T. Power (Eds.) The Handbook of Cognition and Emotion. p. 45-60. Sussex, U.K.: John Wiley \& Sons, Ltd. 1999.

Izard, C.E.. Basic emotions, relations among emotions, and emotion-cognition relations. In: Psychology Review. 99:561-65. 1992.

Jaques, P. A., \& Vicari, R. Considering Student's Emotions in Computer Mediated Learning Environments. In: Z. Ma (Ed). Web-based Intelligent e-Learning Systems: Technologies and Applications (pp.122-138). Hershey, PA: Information Science Publishing. 2005.

Ji, Q. \& Hu, R. 3D Face Pose Estimation and Tracking from a Monocular Camera, Image and Vision Computing, v.20, issue 7, pp.499-511, 2002.

Kapoor, A. \& Picard, R. E. Multimodal Affect Recognition in Learning Environments, ACM MM'05, November 6-11, Singapore. 2005.

Kapur, A., Kapur, A., Virji-Babul, N., Tzanetakis, G. \&. Driessen, P.F. Gesture-Based Affective Computing on Motion Capture Data. In: Proceedings of the International 
Conference on Affective Computing and Intelligent Interaction (ACII) , Beijing, China, October, 2005.

Kshirsagar, S. \& Magnenat-Thalmann, N. A Multilayer Personality Model. In Symposium on Smart Graphics: 107-115. Hawthorne, NY, USA. 2002

Le Doux, J. Emotion: Clues from the Brain. In: Annual Review Psychology. 46:209-235, 1995.

Liao, W., Zhang, W., Zhu, Z., Ji, Q. \& Gray, W. D. Toward a decision-theoretic framework for affect recognition and user assistance. In: International Journal of Human-Computer Studies, 64 (9) pp. 847-873. 2006.

Macedo, A. L. Aprendizagem em ambientes virtuais: o olhar do aluno sobre o próprio aprender. Porto Alegre: UFRGS. Dissertação (Mestrado em Educação), Programa de PósGraduação em Educação, Faculdade de Educação. 2005.

Ortony, A, Clore, G., \& Collins. A. The cognitive structure os emotions. Cambridge: Cambridge University Press, 1988.

Paiva, A. \& Machado, I. Vincent, an autonomous pedagogical agent for on-the-job training. In: Conference on Intelligent Tutoring Systems, 4., San Antonio. Proceedings... Berlin: Springer-Verlag, 1998.

Piaget, J. The relation of affetivity to intelligence in the mental development of the child. In: Bulletin of the Menninger clinic. vol. 26, no 3. 1962.

Piaget, J. Problemas de psicologia genética. In: Os pensadores. São Paulo: Abril Cultural, 1978.

Picard, R. W. Affective Computing. Cambridge: MIT Press. 1997.

Picard, R.W.; Papert, S.; Bender, W.; Blumberg, B.; Breazeal, C.; Cavallo, D.; Machover, T.; Resnick, M.; Roy, D.; Strohecker, C. Affective Learning-A Manifesto. BT Technical Journal, Volume 22, No. 4, pp. 253-269. October, 2004.

Rickel, J. \& Johnson, L. Steve: A Pedagogical Agent for Virtual Reality. In: International Conference on Autonomous Agents, 2. 1998, Minneapolis. Proceedings... New York: ACM Press, p. 165-172. 1998.

Roseman, I.J., Jose, P., \& Spindel, M.S. Appraisals of emotion-eliciting events: Testing a theory of discrete emotions. Journal Personality and Social Psychology, 5(59), 899-915. 1990.

Rousseau, D. \& Hayes-Roth, B.. A social-psychological model for synthetic actors. In: Autonomous Agents 1998, Minneapolis, MN. (p 165-172).1998.

Scherer, K. What are emotions? And how can they be measured? In: Social Science Information 44 (4), 695-729. 2005.

Sloman, A. How many separately evolved emotional beasties live within us? In R.Trappl, P. Petta, and S. Payr, editors, Emotions in Humans and Artifacts, pp. 35-114. MIT Press, Cambridge, MA, 2002.

Shaw, E., Johnson, W.L \& Ganeshan, R. Pedagogical agents on the Web. In Proceedings of the the Third International Conference on Autonomous Agents, ACM Press, NY, 1999, 283-290.

Vicente, A. \& Pain, H. Informing the detection of the students' motivational state: an empirical study. Proceedings of the Sixth International Conference on Intelligent Tutoring Systems, 933-943. 2002.

Vygostki, L.S. Obras Escogidas: Problemas de Psicología General. Madrid: A. Machado Libros. V. 2. 2001.

Vygostki, L.S. A construção do pensamento e da linguagem. São Paulo: Martins Fontes, 2001.

Wallon, H. As origens do caráter na criança: os prelúdios do sentimento de personalidade. São Paulo: Difusão Européia do Livro. 1971.

Zeng, Z.; Ming,J.T.; Zhang, T.; Rizzolo, N.; \& Zhang, Z. Bimodal HCI-related affect recognition, Proc. 6th Int. Conf. on Multimodal Interfaces, pp.137-143, 2004. 